\title{
TECNOLOGIAS E ESTRATÉGIAS NA EDUCAÇÃO A DISTÂNCIA PARA A PROMOÇÃO DA APRENDIZAGEM DE ESTUDANTES COM DEFICIÊNCIA
}

\author{
Lane PRIMO ${ }^{1}$ \\ Vânia Ribas ULBRICHT ${ }^{2}$ \\ Tarcísio VANZIN ${ }^{3}$ \\ Luciane Maria FADEL ${ }^{4}$
}

RESUMO: Este artigo apresenta o resultado de uma revisão sistemática de literatura para identificar as tecnologias utilizadas e as estratégias pedagógicas adotadas para a aprendizagem de estudantes com deficiência. A motivação para este estudo surge da observação do crescimento da participação de estudantes com deficiência em cursos a distância, a projeção de aumento da elaboração de objetos de aprendizagem e de multimídias para serem utilizados nesses cursos que, por conseguinte, requerem cuidados para acessibilidade. As informações levantadas suscitaram reflexões sobre acessibilidade digital e educação, provocaram recomendações e propostas de aprimoramento com vistas à inclusão.

PALAVRAS-CHAVE: Tecnologias de Informação e Comunicação. Estratégias pedagógicas. Deficiência. Acessibilidade. Educação a distância. Revisão sistemática.

\section{Introdução}

A participação de pessoas com deficiência no mercado de trabalho tem se ampliado nos últimos anos, ainda que a passos lentos. O aumento no período de 2012/2013 foi de 8,3\% com a ocupação de 27,5 mil postos de emprego formal (BRASIL, 2014). Em um contexto no qual a inclusão social e o exercício da cidadania são expressões recorrentes na discussão social, ainda há muito a melhorar com vistas a um desenvolvimento sustentável que encontra nas Tecnologias de Informação e Comunicação (TICs) e na Educação duas das características para a economia do conhecimento. Nesse sentido crescem os cursos a distância para formação inicial de trabalhadores, continuada, nível técnico, superior (graduação e pós-graduação) que

\footnotetext{
${ }^{1}$ Pesquisadora de Mídias do Conhecimento do Programa de Pós-Graduação em Engenharia e Gestão do Conhecimento. UFSC - Universidade Federal de Santa Catarina. Florianópolis - SC - Brasil. 88040-900 lane.primo@yahoo.com.br

${ }^{2}$ Líder do Grupo de Pesquisa Núcleo de Acessibilidade Digital e Tecnologias Assistivas. UFSC Universidade Federal de Santa Catarina - Pós-Graduação em Engenharia e Gestão do Conhecimento. Florianópolis - SC - Brasil. 88040-900 - vrulbricht@ gmail.com

${ }^{3}$ Coordenador do Projeto de Pesquisa WebGD Acessível. UFSC - Universidade Federal de Santa Catarina - Pós-Graduação em Engenharia e Gestão do Conhecimento. Florianópolis - SC - Brasil. 88040900 - tvanzin@gmail.com

${ }^{4}$ UFSC - Universidade Federal de Santa Catarina - Pós-Graduação em Design e Expressão Gráfica. Florianópolis - SC - Brasil. 88040-900 - liefadel@gmail.com
} 
matricularam 692.273 estudantes em 2013 (ABED, 2014), configurando-se como oportunidade de desenvolvimento e inserção para pessoas com deficiência.

Em 2013, conforme os dados do Censo EAD.br (ABED, 2014), de 247 instituições de formação que atuam na modalidade a distância, 89 atenderam estudantes com deficiência. As alterações para o atendimento acessível incluíram a produção de videoaulas com legendas, adaptação de ambientes virtuais de aprendizagem (AVAs) e materiais, tradução de material para Braille e Libras, adaptação de layout com acesso via teclado, entre outras. Do mesmo total, 141 instituições produziram e utilizaram objetos de aprendizagem que foram disponibilizados pelo ambiente virtual de aprendizagem. As entidades, em uma visão otimista, projetam para os próximos anos aumentar a produção que inclui, além de objetos de aprendizagem (OAs), o desenvolvimento de animações, simuladores, vídeos, slides, artefatos para realidade aumentada, entre outros a serem utilizados nos cursos. Segundo Macedo (2010, p. 138), em cursos a distância via web a acomodação dos indivíduos com deficiência “[...] condiz com a acessibilidade na web em geral, buscando proporcionar um maior grau de independência ao indivíduo [...]", isto é, as barreiras de acesso podem ser reduzidas no projeto do designer, pelas configurações do sistema operacional, pelo navegador ou pelo uso de tecnologias assistivas. Diante da projeção de produção dos artefatos relatada no censo da ABED, indaga-se se as equipes de desenvolvimento estão preparadas para que os mesmos sejam acessíveis, assim como se os educadores (professores e tutores) estão preparados para o uso dos OAs com a finalidade de mediação dos processos visando à aprendizagem de todos.

Por acreditar que a educação a distância potencializa o desenvolvimento de estudantes com deficiência para que possam exercer sua cidadania e também serem inseridos no mundo do trabalho, buscou-se na revisão sistemática da literatura (SAMPAIO; MANCINI, 2007; ULBRICHT et al., 2012; OBREGON; VANZIN; ULBRICHT, 2015) subsídios que possam auxiliar a compreender o estado atual e contribuir de alguma maneira para a melhoria da educação inclusiva.

\section{Procedimentos metodológicos da pesquisa}

A pesquisa foi realizada de acordo com as seguintes etapas: (1) definição dos objetivos da investigação; (2) elaboração da questão da pesquisa; (3) definição dos parâmetros da pesquisa; (4) análise dos documentos e (5) síntese dos resultados. 


\section{Objetivos da investigação}

A revisão sistemática pretende atingir os seguintes objetivos: (1) mapear as pesquisas sobre tecnologias utilizadas na educação a distância para estudantes com deficiência; (2) identificar as tecnologias que apresentam resultados eficazes; (3) identificar estratégias metodológicas aplicadas para a aprendizagem dos estudantes com deficiência; e, por fim, (4) levantar as tecnologias que utilizam mídias e relacioná-las às recomendações de acessibilidade.

\section{Definição da questão da pesquisa}

A partir da problematização e dos objetivos a serem atingidos, a investigação procura responder ao seguinte questionamento: Quais as tecnologias e estratégias pedagógicas utilizadas para promover a aprendizagem de estudantes com deficiência na educação a distância?

\section{Parâmetros da pesquisa}

A partir do questionamento, a pesquisa inicial foi realizada no Portal de Periódicos CAPES, utilizando a busca por assunto. A escolha do portal deve-se ao fato de reunir 126 bases referenciais, além das vantagens de agilizar o processo de investigação e de disponibilizar o acesso a um número maior de documentos completos. A busca foi realizada utilizando-se os seguintes parâmetros: a) Assunto/termo de busca: (1) "educação a distância AND deficiência" e (2) "objeto + aprendizagem AND acessibilidade"; b) Para as duas buscas, o refinamento visando à delimitação do assunto foi realizado por meio de filtros; c) O período selecionado foi dos últimos cinco anos - 2010 a 2015.

Ao escolher os termos "educação a distância", "deficiência", "objeto de aprendizagem" e "acessibilidade" pretende-se verificar se há trabalhos realizados no Brasil. Considera-se importante conhecer a realidade brasileira, uma vez que foi apontado o atendimento à estudantes com deficiência assim como a projeção de ampliação do desenvolvimento de objetos de aprendizagem no CensoEAD.br (ABED, 2014).

A primeira busca referente aos assuntos "educação a distância AND deficiência" apresentou 125 documentos. No refinamento, os seguintes termos foram marcados no filtro como critério válido de seleção "Educação Especial, Special Education, Distance Education, Educação a Distância, Deafness, Surdez, Visual Disability” reduzindo para 16 documentos. Foram excluídos 109 documentos que continham os termos "Children, Social Sciences, Avaliação, Evaluation, Brazil, Women, Child Health, Health, Educação 
Indígena, Indigenous Educational, Poverty, Inclusion, Ageing”. A segunda busca referente aos assuntos "objeto + aprendizagem AND acessibilidade" apresentou três documentos. De igual maneira, no refinamento, permaneceram os termos "Learning Object, Visual Impairment, Accessibility Inclusion, Scrapbook, Learning Objects, OBAA Standards, Ontologia, Inclusive Education, Accessibility, Matemática, Mathematics, Acessibilidade, Science General, Mediation Strategies, Deficiência Visual, Science (General), Inclusão Escolar, Ontology, Estratégia”. Nenhum documento foi excluído.

A leitura dos resumos com o fito de identificar termos que pudessem contribuir para responder ao questionamento resultou na seleção de quatro documentos para os assuntos "educação a distância AND deficiência" que apresentaram os termos "Web, metodologias, práticas pedagógicas, WAI, W3C, relato de experiência" - 12 foram excluídos. Para os assuntos "objeto + aprendizagem AND acessibilidade", a seleção foi de dois documentos que apresentaram os termos "WAI, W3C, Recursos de acessibilidade" - 1 (um) foi excluído. Portanto, 6 (seis) documentos no total resultante da busca no portal de periódicos CAPES.

Para garantir ampla cobertura, a busca foi repetida nas bases Scielo, Scopus e Web of Science, o que resultou no acréscimo de mais um artigo com as palavras-chave “educação a distância AND deficiência”, totalizando 7 (sete) documentos.

Os tipos dos documentos selecionados foram 3 (três) artigos, 1 (uma) tese e 1 (uma) dissertação para a pesquisa sobre "educação a distância AND deficiência"; 1 (um) artigo e 1 (uma) tese para a pesquisa sobre "objeto + aprendizagem AND acessibilidade".

Após a leitura dos 7 (sete) documentos, foram selecionados 4 (quatro) que apresentaram pontos a serem mapeados, compilados e analisados na busca da resposta ao questionamento. Segundo o tipo, para "educação a distância AND deficiência” foram 1 (um) artigo e 1 (uma) dissertação; para "objeto + aprendizagem AND acessibilidade" foram 1 (um) artigo e 1 (uma) tese.

Vale observar que, na pesquisa realizada utilizando o filtro dos termos “educação a distância AND deficiência”, foram encontrados artigo e dissertação da mesma autora versando sobre o mesmo assunto. Após uma rápida análise, a opção foi pela dissertação porque a mesma apresentava detalhes que respondiam de forma mais precisa ao tema em estudo. 


\section{Análise dos documentos}

Os passos adotados para a análise dos documentos foram: (1) criação de quadro auxiliar contendo as categorias eleitas como chave, a saber: tecnologias utilizadas, estratégias pedagógicas adotadas, considerações (da pesquisadora), deficiências que participaram das experiências, potencialidades e fragilidades para serem preenchidas por documento lido; (2) agrupamento dos dados para a compilação e síntese das informações - Tabela 1.

\section{Síntese dos resultados}

Os parágrafos que seguem mostram os resultados da compilação:

O primeiro deles é o agrupamento por área que mostra as palavras-chave atribuídas pelos autores e, por fim, as mais citadas cujos numerais indicam repetições. A identificação da área foi feita pelas informações dos documentos. $\mathrm{Na}$ área "Informática", as palavras-chave atribuídas pelos autores foram "estratégia de mediação, ontologia, Matemática, objetos de aprendizagem, deficiência visual e padrão OBAA"; nas áreas "Matemática, Estatística, Tecnologia", as palavras foram "objeto de aprendizagem, Scrapbook, acessibilidade e inclusão"; na área "Educação especial", as palavras foram "tecnologias de informação e comunicação, inclusão educacional e objeto de aprendizagem" e na área "Informática na Educação Especial", as palavras foram "educação especial, inclusão digital, acessibilidade, tecnologia educacional e educação a distância". As mais citadas foram "inclusão (digital, educacional)" e "objeto (s) de aprendizagem" - 3 (três) ocorrências cada; "acessibilidade" e "tecnologia (s) (educacional, de informação e comunicação)" - 2 (duas) ocorrências cada.

À luz dos objetivos e tendo o questionamento como lente para a leitura dos documentos, as informações de destaque foram preenchidas nos quadros. A Tabela 1 apresenta o resultado da compilação. 
Tabela 1 - Revisão Sistemática da Literatura - Compilação

\begin{tabular}{|l|l|}
\hline $\begin{array}{l}\text { Tecnologia } \\
\text { utilizada }\end{array}$ & $\begin{array}{l}\text { AVA inclusivo (destaque para barra de acessibilidade e blog) }-1 \\
\text { Objetos de aprendizagem }-3\end{array}$ \\
\hline $\begin{array}{l}\text { Estratégia } \\
\text { pedagógica } \\
\text { adotada }\end{array}$ & $\begin{array}{l}\text { Baseada em: } \\
\text { Comunicação }-4 \\
\text { Interação social da diferença fundamentada em Vygotsky }-2 \\
\text { Conceito da Zona de Desenvolvimento Proximal (ZDP }=\text { diferença entre nível } \\
\text { de desenvolvimento real e nível de desenvolvimento potencial) }-1 \\
\text { Construcionismo - 1 }\end{array}$ \\
\hline $\begin{array}{l}\text { Experiências } \\
\text { aplicadas }\end{array}$ & $\begin{array}{l}\text { Deficiência visual }-2 \\
\text { Síndrome de Down }-2 \\
\text { Deficiência auditiva }-1 \\
\text { Estudantes desinteressados }-1\end{array}$ \\
\hline $\begin{array}{l}\text { Potencialidades } \\
\text { Autonomia do estudante }-4 \\
\text { Respeito às diferenças e individualidades }-3 \\
\text { Uso de multimídias }-4 \\
\text { Redução do uso de tecnologias assistivas (substituição pelo digital) }-1\end{array}$ \\
\hline $\begin{array}{l}\text { Fragilidades } \\
\text { (requer } \\
\text { melhorias) }\end{array}$ & $\begin{array}{l}\text { Navegabilidade (muitas janelas - desorientação) }-1 \\
\text { Leitores de tela (não identificam caixas de verificação e botões de opção) }-1 \\
\text { Uso operacional (conhecimento inserir, gravar arquivos) }-1 \\
\text { Qualidade descritiva das narrações }-1 \\
\text { Inserção de Libras em todos os elementos necessários }-1 \\
\text { Limites do Flash para web e dispositivos móveis }-1\end{array}$ \\
\hline
\end{tabular}

Fonte: Elaboração própria.

\section{Discussão}

O mapeamento das pesquisas sobre as tecnologias e estratégias utilizadas na educação a distância voltadas para estudantes com deficiência revelou um quantitativo de trabalhos selecionados pequeno para o foco da pesquisa, considerando o número de 125 itens que apareceram na busca por assunto "educação a distância" e "deficiência". Esses termos estavam relacionados aos campos social, político e infraestrutura. Dos trabalhos selecionados, a concentração da produção científica relacionada ao tema foi das regiões Sudeste e Sul. Com relação ao período, constata-se que o tema passa por crescimento e amadurecimento da questão - um de 2010, um de 2011 e dois de 2012.

As tecnologias relacionadas - ambiente virtual acessível e os objetos de aprendizagem (OAs) mostraram resultados positivos nos relatos. O objeto de aprendizagem Scrapbook foi tema de estudo de dois trabalhos (JEREZ et al., 2011; BARDY, 2010). Em um deles, foi proposto o desenvolvimento para as plataformas mobile e web e para sistemas operacionais Linux e Mac OS X (JEREZ et al., 2011) - o que configura como uma ampliação de acesso. Considera-se um passo importante para a interoperabilidade, 
porém os dispositivos móveis ainda precisam de melhorias a serem implementadas para que as pessoas com deficiência possam usufruir dos recursos básicos de forma plena.

A Matemática foi a disciplina que impulsionou a criação de OAs nos trabalhos analisados. Em comum, no desenvolvimento foram citadas as recomendações W3C, WCGA 2.0 (JEREZ et al., 2011; LOPES, 2012), porém não foram citados se houve algum tipo de verificação utilizando os validadores de acessibilidade do W3C. Em Lopes (2012), o E-MAG 3.0 foi utilizado como parâmetro. Convém alertar que os objetos de aprendizagem foram elaborados em Flash e alguns foram refeitos em HTML. A autora justificou a escolha do Flash por causa da familiaridade dos bolsistas com este software e suas ferramentas de acessibilidade, porém vale ressaltar que nessa época já se anunciava o fim do Flash pela baixa interoperabilidade e adaptabilidade. Com isso há o comprometimento da continuidade do objeto uma vez que não é suportado nos sistemas operacionais Android e IOS. O Desenho universal apareceu em Jerez et al. (2011). Um dos trabalhos citou o padrão OBAA (Objetos de aprendizagem baseados em agentes) para a elaboração do OA indicado como ideal para TVD, Web e mobile (LOPES, 2012). Um estudo de Silva, Vicari e Passerino (2014) mostraram que os metadados do padrão OBAA apontaram maior potencial de uso comparados a especificações Dublin Core e IEEE LOM por contemplar as tecnologias emergentes e apresentar aspectos sociais por meio de uma categoria de acessibilidade.

Todos os trabalhos sinalizaram a utilização de estratégias pedagógicas voltadas para a comunicação (professor-estudante, professor-grupo de estudantes, estudantes entre si) por meio de atividades ou situações em que havia troca de informações. Santarosa, Conforto e Basso (2012) utilizaram ferramentas fórum, chat, blog, entre outras, e reportaram como positivo os processos de interação e de expressão dos participantes de forma autônoma. Com relação ao aspecto comunicação, dois trabalhos ressaltaram a interação social histórica de Vygotsky como apropriada para o desenvolvimento de estudantes com deficiência (SANTAROSA; CONFORTO; BASSO, 2012; LOPES, 2012). Em Bardy (2010, p. 106), os objetos de aprendizagem foram elaborados na perspectiva do Construcionismo ${ }^{5}$ de forma que o estudante "tenha

${ }^{5}$ Construtivismo - teoria pedagógica postulada por Piaget em que o estudante é o sujeito no processo de aprendizagem, no qual o conhecimento é construído a partir de suas experiências; o Construcionismo deriva do construtivismo, teoria desenvolvida por Seymor Papert, e aparece nas discussões relacionadas à Informática na Educação. A construção do conhecimento se dá pela interação do estudante com o objeto (computador) ligado a algo do seu interesse, há uma motivação que cria um significado. Neste sentido, difere do Instrucionismo porque nessa abordagem o computador é utilizado como uma máquina de ensinar - as ações são programadas e previsíveis. 
a oportunidade de construir o conhecimento por meio de atividades práticas que o levem a refletir suas ações" e mais "de maneira lúdica e significativa". O Construcionismo e o papel da mediação é corroborado no trabalho de Schwabe (2013, p.774, tradução nossa). Das observações conclui que o paradigma construcionista é um "método adequado para os nativos digitais".

Os trabalhos selecionados relataram pontos positivos, mas também reconheceram que há a necessidade de aprofundar mais os estudos, de rever o planejamento das aulas para incluir o uso dos objetos de aprendizagem, assim como providenciar as adaptações das estratégias metodológicas para estudantes com deficiência. Em Bardy (2010), foi relatado que a intervenção do professor é necessária para instrução, mediação, ajuste no atendimento aos estudantes com deficiência e que os docentes precisam de capacitação para atuar tanto com as tecnologias quanto com a situação das deficiências. A mediação, segundo Lopes (2012), requer ações que visem o desenvolvimento dos sujeitos, baseado na teoria sócio-histórica, que utiliza o conceito de Zona de Desenvolvimento Proximal (ZDP) e trabalha a diferença dos processos do Nível de Desenvolvimento Potencial (NDP) e Nível de Desenvolvimento Real (NDR). No processo para o desenvolvimento, a mediação ocorre entre professor-estudante, professor-OA, estudante-OA e professor-estudante-OA.

Os trabalhos defendem a contribuição das tecnologias para a aprendizagem. Santarosa, Conforto e Basso (2012) destacaram a construção de conhecimento de forma colaborativa e a aprendizagem centrada no estudante com ou sem deficiência por meio das ferramentas de autoria e colaboração desenvolvidas. Esses autores referenciaram validações realizadas em trabalhos de outros pesquisadores nos quais participaram pessoas com limitação visual, jovens com síndrome de Down, pessoas com TDAH ${ }^{6}$, surdos e pessoas com paralisia cerebral (SONZA, 2008; MACHADO, 2007; BOIASKI, 2007; BARTH, 2008; GOES, 2009; LIMA, 2010; CUNHA, 2008 apud SANTAROSA; CONFORTO; BASSO, 2012). A síndrome de Down também foi relatada em Bardy (2010) no depoimento de uma das coordenadoras que acompanhou a atuação de um docente em uma turma. No relato, a estudante observada mostrou-se mais motivada nas aulas em que os objetos de aprendizagem foram utilizados e na sala de aula foram constatadas melhoras no convívio e no aprendizado. Os estudantes que demonstravam desinteresse também melhoraram suas performances e se engajaram mais nas atividades

${ }^{6}$ Transtorno do Déficit de Atenção e Hiperatividade. 
com o uso dos objetos de aprendizagem. Lopes (2012) concluiu que o uso dos OAs no processo de mediação (estudantes, objeto, professor) possibilitou aos participantes estudantes com baixa visão, o desenvolvimento dos conceitos matemáticos por meio da linguagem e o convívio social. Entretanto, afirmou que há muitos desafios a serem vencidos pelos professores para melhorar as práticas, a apropriação do conhecimento do uso de recursos educacionais e de como lidar com as deficiências.

\section{Acessibilidade Digital e Educação - Convergências e Perspectivas}

Os resultados apresentados suscitam reflexões sobre a acessibilidade digital e a educação a serem discutidas nos próximos parágrafos.

Em Lopes (2012, p.54), a acessibilidade digital teve “[...] a finalidade de permitir o acesso a diversos tipos de usuários aos conteúdos digitais, proporcionando igualdade de condições e de inclusão.” Para Santarosa, Conforto e Basso (2012, p. 452), a acessibilidade teve como foco a ampliação dos “[...] recursos para potencializar a interação, o desenvolvimento e a ação sociocognitiva." No trabalho de Jerez et al. (2011, p. 102) a acessibilidade dos objetos de aprendizagem se efetiva quando "[...] todos os usuários têm acesso às mesmas funcionalidades disponíveis no sistema, sem que ocorra qualquer distinção ou privilégio." Para o termo acessibilidade, as linhas de pensamento e intenções traduzem igualdade, comunicação, desenvolvimento cognitivo e social. Contudo para que a acessibilidade digital aconteça, os desenvolvedores precisam conhecer mais profundamente e observar as recomendações para a elaboração de objetos de aprendizagem acessíveis.

Bardy (2010, p.146) relacionou que os aspectos mais importantes da experiência de OAs, em turmas que contou com a participação de estudantes com deficiência, foram a maior interação entre os eles, o interesse na realização das atividades, os momentos de ludicidade que tornaram o aprendizado mais prazeroso, assim como terem estimulado os estudantes para a aprendizagem. No relato dos professores, os OAs foram considerados “[...] importantes para o processo de ensino-aprendizagem de todos os alunos, sejam

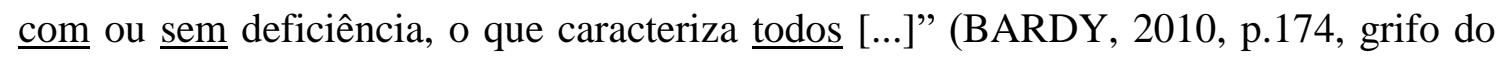
autor). A citação expressa de forma subjetiva as intenções elencadas no parágrafo anterior, porém acredita-se que indicadores qualitativos e quantitativos podem ajudar a melhorar os processos. 
As visões de acessibilidade aplicadas às soluções dos trabalhos citados mostram que diversas iniciativas estão sendo realizadas para superar o desafio de criar tecnologias que priorizem e "[...] minimizem as dificuldades que ainda permeiam o acesso, a navegação e a comunicação entre usuários [...]” (OBREGON; FLORES, 2011, p.64). As implementações tecnológicas, estratégias educacionais e sociais ajudaram a elevar os resultados das experiências relatadas com relação a maior autonomia dos estudantes, respeito às diferenças e individualidades (SANTAROSA; CONFORTO; BASSO, 2012; LOPES, 2012; JEREZ et al., 2011; BARDY, 2010) e substituição do uso de tecnologias assistivas pela digital (SANTAROSA; CONFORTO; BASSO, 2012).

O uso das multimídias apresentadas nos trabalhos, apesar destas terem sido potenciais para o desenvolvimento dos estudantes com deficiência, ainda precisa de aprimoramento em diversos aspectos para que elas se tornem plenamente acessíveis. Relatos sobre questões de usabilidade, tais como dificuldades de navegação e desorientação e impossibilidade de programas leitores de tela identificarem alguns dos componentes dos OAs, configuram algumas das fragilidades citadas que estão relacionadas às experiências operacionais. Outros pontos frágeis que precisam de melhorias citados foram: qualidade descritiva das narrações, inserção de Libras em elementos que requerem esclarecimentos, audiodescrição de imagens ou situações; esses pontos interferem nas experiências cognitivas dos estudantes com deficiência. Tais fragilidades denotam que os princípios perceptível, operável, compreensível e robusto das recomendações de acessibilidade do WCAG 2.0 não foram ou foram parcialmente atendidas. A diversificação de recomendações citadas pelos autores (um dos trabalhos utilizou como base W3C, WCGA 2.0, outro E-MAG e outro o Desenho Universal) demonstra a necessidade de uma convergência.

Sobre essa questão, Macedo (2010) apresentou um levantamento acerca dos esforços para a harmonização dos padrões por parte de várias entidades com vistas a reduzir a fragmentação, as divergências e os conflitos. Após estudos, Macedo (2010) elaborou uma proposta de Diretrizes de Acessibilidade em Objetos de Aprendizagem, fruto da convergência das principais recomendações - Princípios de Design Universal (para conteúdo web) + Recomendações de Acessibilidade para Criação de Conteúdo Online (W3C-WCAG 2.0 e W3C-WCAG 1.0) + IMS-ACC Guide. As recomendações das Diretrizes criadas são para a disponibilização de opções alternativas a fim de tornar as mídias (imagens estáticas, imagens em movimento, tabelas, textos, áudio e gráficos) que compõem os objetos de aprendizagem acessíveis. Isto significa disponibilizar conteúdo 
equivalente ou alternativo ${ }^{7}$. As Diretrizes de Acessibilidade em Objetos de Aprendizagem podem contribuir para a redução das fragilidades apresentadas.

\section{Considerações finais}

Este artigo apresentou uma revisão sistemática de literatura para verificar quais tecnologias estão sendo utilizadas e quais são as estratégias pedagógicas adotadas visando o sucesso da aprendizagem de estudantes com deficiência. A motivação para este estudo partiu da observação do crescimento da participação de estudantes com deficiência em cursos da educação a distância, a projeção de aumento da elaboração de objetos de aprendizagem e de multimídias para serem utilizados nesses cursos que, por conseguinte, requerem cuidados para acessibilidade.

A metodologia de revisão sistemática de literatura fez emergir informações sobre tecnologias, estratégias pedagógicas, deficiências atendidas, potencialidades e fragilidades na educação a distância envolvendo estudantes com deficiência no período de 2010 a 2015.

As palavras-chave dos trabalhos analisados mostram que inclusão digital e social, objeto de aprendizagem, acessibilidade, tecnologia educacional e de informação e comunicação são as mais relevantes para este estudo e podem se configurar como parâmetro para futuras pesquisas.

As deficiências que tiveram estudantes participantes foram visual, síndrome de Down e auditiva.

O estudo revelou que ainda são poucos os trabalhos sobre tecnologias na educação a distância voltados para estudantes com deficiência. Identificou o uso de um ambiente virtual acessível e de objetos de aprendizagem que foram aplicados em situações didáticas mediadas por professores. Verificou que as estratégias pedagógicas mais utilizadas são as que promovem a comunicação entre os participantes. As correntes pedagógicas utilizadas foram a interação social da diferença fundamentada em Vygotsky e o construcionismo de Papert. Identificou que apesar do atendimento às recomendações para acessibilidade, tanto o ambiente virtual quanto os OAs precisam de melhorias para que o desenvolvimento da aprendizagem dos estudantes seja bem-

${ }^{7}$ Conteúdo equivalente é idêntico ao que deu origem, porém é fornecido em uma modalidade diferente. Exemplo: texto original pode ser disponibilizado pelos equivalentes em áudio e texto para impressão em Braille; conteúdo alternativo possui o mesmo objetivo final de aprendizagem, porém é apresentado de outra forma ou formato, é uma ampliação do conteúdo equivalente (MACEDO, 2010, p.169). 
sucedido. Verificou que tanto desenvolvedores quanto docentes precisam de capacitação para poder melhorar processos de trabalho no aspecto da usabilidade e das estratégias pedagógicas para lidar com os OAs e as situações em que participem estudantes com deficiência.

As limitações desta revisão estão na busca por trabalhos nacionais e de um intervalo temporal a partir de 2010 por considerar que o avanço tecnológico e da educação a distância foi mais intenso nesse período no Brasil. Esta opção teve como intenção conhecer a realidade brasileira. De forma alguma invalida contribuições que porventura possam ter ficado fora do escopo.

Recomenda-se como proposta:

- realizar pesquisas com o foco no acompanhamento do uso de OAs acessíveis e recursos educacionais que tenham sido elaborados por desenvolvedores ou educadores, obedecendo às Diretrizes de Acessibilidade para Objetos de Aprendizagem (MACEDO, 2010) a fim de avaliar a efetividade. A sugestão é de estudos comparativos da percepção do estudante com deficiência e do educador (tutor ou professor) que acompanha o processo de ensinoaprendizagem;

- elaborar critérios e indicadores para avaliar as estratégias pedagógicas desenvolvidas em conjunto com os OAs acessíveis para diversos tipos de deficiência;

- promover a qualificação de desenvolvedores e educadores para a elaboração de objetos de aprendizagem e recursos educacionais de acordo com as Diretrizes de Acessibilidade de Objetos de Aprendizagem desenvolvida por Macedo (2010);

- divulgar os resultados na forma de artigos para a disseminação do conhecimento.

As vantagens da Educação a Distância e as tecnologias envolvidas são comprovadas por diversos estudos. Para pessoas com deficiência são caminhos para a inclusão social e inserção no mercado de trabalho. Enquanto pesquisadores o nosso papel é de suma importância como agentes de promoção desta inclusão. 


\section{TECHNOLOGIES AND STRATEGIES IN DISTANCE EDUCATION FOR STUDENTS WITH DISABILITIES LEARN}

ABSTRACT: This paper presents the results of a systematic review of the literature to identify the technologies used and the pedagogical strategies adopted for students with disabilities. The motivation for this study arises from the observation of the growth of the participation of students with disabilities in distance learning courses, the projection of increased development of learning objects and multimedia for use in these courses, therefore, requiring care for accessibility. The information gathered reveal thoughts on digital accessibility and education and have resulted in recommendations and proposals with a view to enhancing inclusion.

KEY WORDS: Information and Comunication Technologies. Pedagogical strategies. Disabilities. Accessibility. Distance learning. Systematic review.

\section{REFERÊNCIAS}

ASSOCIAÇÃO BRASILEIRA DE EDUCAÇÃO A DISTÂNCIA [ABED]. Censo

EAD.br: relatório analítico da aprendizagem a distância no Brasil $2013=$ Censo EAD.br: analytic report of distance learning in Brazil. Tradução de Maria Thereza Moss de Abreu. Curitiba: Ibpex, 2014. Disponível em: <http://www.abed.org.br/site/pt/midiateca/ censo_ead/1272/2014/10/censoead.br_2013/2014>. Acesso em: 20 abr. 2015.

BARDY, L. R. Objetos de aprendizagem em contextos inclusivos: subsídios para a formação de professores. 2010. 225f. Dissertação (Mestrado em Educação Especial)Universidade Federal de São Carlos, São Carlos, 2010. Disponível em:

$<$ http://www.bdtd.ufscar.br/htdocs/tedeSimplificado//tde_busca/arquivo.php?codArquiv o=3150>. Acesso em: 9 abr. 2015.

BRASIL. Secretaria de Direitos Humanos da Presidência da República. Cresce em 8,3\% o número de pessoas com deficiência com contrato formal de trabalho. Brasília, nov. 2014. Disponível em:

$<$ http://www.sdh.gov.br/noticias/2014/novembro/cresce-em-8-3-o-numero-de-pessoascom-deficiencia-com-contrato-formal-de-trabalho>. Acesso: 20 abr. 2015.

JEREZ, M. O. et al. Aspectos tecnológicos no desenvolvimento de um objeto de aprendizagem com acessibilidade: a experiência com o Scrapbook. Colloquium Exactarum, Presidente Prudente, v.3, n.2, p.93-104, 2011. Disponível em: <http://revistas.unoeste.br/revistas/ojs/index.php/ce/article/viewArticle/714>. Acesso: 9 abr. 2015.

LOPES, A. M. A. Estratégias de mediação para o ensino de matemática com objetos de aprendizagem acessíveis: um estudo de caso com alunos com deficiência visual. 2012. 290f. Tese (Doutorado em Informática na Educação)-Centro de Estudos Interdisciplinares em Novas Tecnologias da Educação, Universidade Federal do Rio Grande do Sul, Porto Alegre, 2012. Disponível em: <http://www.lume.ufrgs.br/handle/10183/55685>. Acesso em: 9 abr. 2015. 
MACEDO, C. M. S. Diretrizes para criação de objetos de aprendizagem acessíveis. 2010. 271f. Tese (Doutorado em Engenharia e Gestão do Conhecimento)-Universidade Federal de Santa Catarina, Florianópolis, 2010. Disponível em:

<http://www.egov.ufsc.br/portal/sites/default/files/claudia-mara-scudelari-demacedo.pdf $>$. Acesso em: 10 mar. 2015.

OBREGON, R. F. A.; FLORES, A. R. B. Educação inclusiva: criando e recriando possibilidades para o compartilhamento de conhecimento em Ambiente Virtual de Ensino e Aprendizagem. In: ULBRICHT, V.; VANZIN, T.; VILLAROUCO, V. Ambiente virtual de aprendizagem inclusivo. Florianópolis: Pandion, 2011. p.49-68.

OBREGON, R. F. A.; VANZIN, T.; ULBRICHT, V. R. Revisão sistemática de literatura processos de aprendizagem das pessoas surdas ou com deficiência auditiva e pessoas cegas ou com deficiência visual. In: . AVA inclusivo: recomendações para design instrucional na perspectiva da alteridade. São Paulo: Pimenta Cultural, 2015. Cap. 5.

SAMPAIO, R. F.; MANCINI, M. C. Estudos de revisão sistemática: um guia para síntese criteriosa da evidência científica. Revista Brasileira de Fisioterapia, São Carlos, v.11, n.1, p.83-89, 2007. Disponível em: <http://www.scielo.br/pdf/rbfis/v11n1/12>. Acesso em: 10 mar. 2015.

SANTAROSA, L. M. C.; CONFORTO, D.; BASSO, L. O. Eduquito: ferramentas de autoria e de colaboração acessíveis na perspectiva da web 2.0. Revista Brasileira de Educação Especial, Marília, v.18, n.3, p.449-468, 2012. Disponível em: <http://www.scielo.br/pdf/rbee/v18n3/a07.pdf>. Acesso em: 9 abr. 2015.

SCHWABE, R. H. Las tecnologías educativas bajo u paradigma construccionista: un modelo de aprendizaje en el contexto de los nativos digitales. Revista IberoAmericana de Estudos em Educação, Araraquara, v.8. n.3, p.738-746, 2013. Disponível em: <http://seer.fclar.unesp.br/iberoamericana/article/view/6596/4853>. Acesso em: 13 set. 2015.

SILVA, J. M. C.; VICARI, R. M.; PASSERINO, L. M. Análise técnica e pedagógica de metadados para objetos de aprendizagem. In: DUQUE MÉNDEZ, N. D.; CARRANZA, D. O.; CADAVID, J. M. Objetos de aprendizaje, repositorios y federaciones... conocimiento para todos. Manizales: Universidad Nacional de Colombia, 2014. Cap.2.

ULBRICHT, V. R. et al. Visualização do conhecimento: revisão sistemática da literatura e mapas conceituais. Revista Educaonline, Rio de Janeiro, v.6, p.94-113, 2012. Disponível em:

$<\mathrm{http}: / / \mathrm{www}$. latec.ufrj.br/revistas/index.php?journal=educaonline \&page=article \&op=vi ew\&path\%5B\%5D=307>. Acesso em: 14 mar. 2015. 